\title{
Research on computer simulation theory and experimental verification technology of the working process of automobile airbag
}

\author{
Baogui Zuo \\ Automotive Engineering institute, Jiangxi University of Technology, Nanchang 330098, China
}

Keywords: Automobile airbag; Computer simulation theory; Experimental verification technology

\begin{abstract}
As a convenient modern means of transportation, the car has brought great convenience to people's life. But at the same time, the traffic accidents cause serious losses of people's lives and property. Since the eighteenth century when the car was born, it has been accompanied by vehicle safety problems. In order to improve vehicle safety, the developed countries formulated and implemented relevant laws and regulations. The automobile safety can be divided into active safety and passive safety. Active safety refers to the ability of the car to prevent car accidents; passive safety refers to the ability of the car to protect passengers and pedestrians when accidents occur. Vehicle passive safety relates to the structure strength of the body, interior softening, energy-absorbing steering column, occupant restraint system, interior fire resistance and human biomechanical characteristics and other related contents, which can be divided into two aspects of work: the reduction of damage in collision and the first aid after collision. The test detection technology proposed in this paper conducts working performance tests for the airbag to simulate the supplementary restraint system. By comparing and analyzing the curves of the simulation results and the experimental results, the validity of the airbag simulation algorithm is verified. The simulation algorithm considers the leakage of the gas inside the airbag, which will make the simulation results closer to the experimental results.
\end{abstract}

\section{Introduction}

Since the first automobile regulations, each new car regulations, especially the formulation and implementation of the safety regulations, will put forward new requirements for the design of automobile. In the control of these regulations, in order to improve the competitiveness of products, major car manufacturers and research institutions carry out special researches on vehicle safety. Vehicle safety research gradually separates from other research fields of automotive technology to form an independent branch. Among them, the first aid after the collision includes passengers rescuing themselves and people outside the vehicle providing emergency rescue for the passengers. To reduce the damage of collision, the following six aspects are included:

(1) Increase the living space after the collision, the main measures including raising the roof strength, side door strength and door lock.

(2) Reduce the damage of a second collision to the occupants. The main measures include: install safety belts; improve the fixation strength of seat belt; install airbag; use energy-absorbing plastic bumper, collapsible energy-absorbing steering wheel and cushion for knees etc. In addition, car interiors softening, the dashboard softening and fixing the window glass firmly and avoiding the 
damage of the windshield debris are effective measures to reduce the damage of a second collision to the occupants.

(3) Prevent passengers from being thrown out. The main measures include enhancing the strength of door lock, the firmness of the door hinge, the firmness of the safety belt, the firmness of the seat and the strength of the vehicle body, etc..

(4) Prevent fire. The main measures include improving the fire resistance of vehicle interior decoration materials, anti-leakage of the fuel system, fireproof of the electrical systems.

(5) Be convenient for passengers to escape and be rescued. The main measures include setting safety exit, and making the window easy to be opened but uneasy to be deformed.

(6) Prevent the pedestrians from being hit. The main measures include improving the driver's vision and optimizing the design of the protrusion on the body, energy-absorbing bumper, decoration trim and driving stability. In the research of vehicle collision passive safety, the contents of passive safety are divided into the following three parts: investigation and analysis of traffic accidents, the collision biomechanical study and the safety countermeasures research.

\section{Computer simulation of the working process of the vehicle airbag}

Because the collision is a transient process, although the test method can solve some problems in research and design, there are still many issues that cannot be resolved. With the development of computer technology and computer simulation technology, a lot of problems in the research and design of the airbag can be solved with the help of computer simulation technology. Application of computer simulation technology in this area can be divided into two levels: the first level is the simulation of the unfolding process of the airbag in given characteristics and the affecting process of the collision of human body and other structures inside the vehicle; the second level is the simulation of the collision of the vehicle with the airbag and the model.

Although the computer simulation technology has yet to be improved, it has become an indispensable tool of airbag. As for the application of the first level mentioned above, it can help optimize the airbag structure form, folding form, shape and size and installation. Its most important advantage is that it cannot only simulate the working process of the airbag in any conditions, but also can provide any concrete and specific data of the airbag working process, which is hard to achieved by the tests. Fig. 1 shows the state diagram for the collision of a human model and airbag obtained by using computer simulation technology. For the application of the second level, the computer simulation technology has an effect that is irreplaceable by experimental technology.

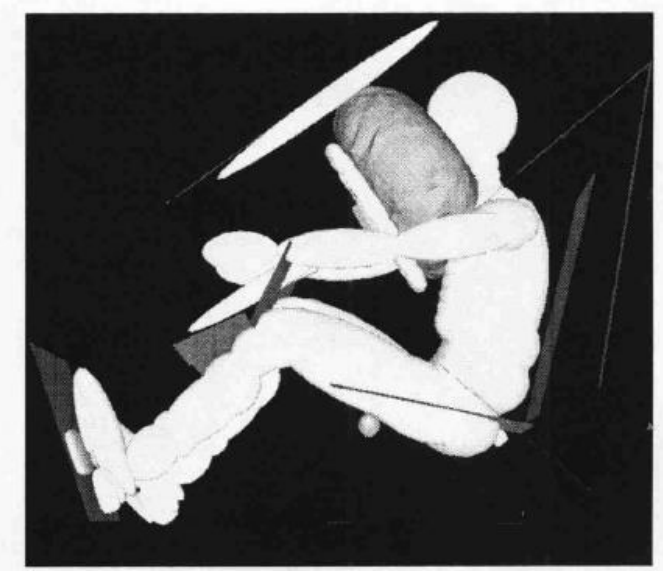

Fig. 1 the state diagram for the collision of a human model and airbag 
DYNA3D is used to study this problem, which has the following characteristics:

(1) The finite element model of dummy: it includes 5,270 solid elements, 530 shell elements and 264 beam elements, using the material model including models with elastic, viscoelastic, and foam material.

(2) Airbag model: it is expressed with hierarchical elastic film force unit without crumple; material model introduces the model with fiber, the isotropic elastic layer, orthotropic and smearing crumple; it also considers the airbag folding algorithm.

(3) Contact model: it considers the mutual contact of air bag, the relevant parts of the body and internal geometry in the body, and the contact processing mainly adopts the Hallqulst to be applied to the simple surface contact algorithm (included in DYNA3D) of the shell structure analysis.

(4) Simplified model: assuming the pressure, density and temperature in the airbag are uniform distribution. Third, the Simon connected finite element simulation with structure design of airbag in 1997.

The finite element model of the airbag is established through ZD or 3DCAD model of the airbag. To establish the finite element model of folding or unfolding airbag and accurately simulate the shape of airbag in the course of unfolding is a complicated project. At present, there are mainly two kinds of modeling methods.

1. Direct folding method

The direct folding method modeling is to establish the corresponding finite element grid according to the actual method of folding airbag. Driver side airbag can be classified as ZD airbag, because it is composed of two circular planes and can easily be flatten, using the direct folding method. Take the driver side airbag as an example. The folding pattern is shown in Fig. 2, the modeling procedure is:

(1) Establish the unfolded geometric shape (surface) according with the size of the airbag; the driver side airbag is two round planes; the gas vent cannot be considered but the shape of the spray hole of the gas generator must be considered;

(2) Determine the folding line according to the actual folding method; the folding line divides the two round plane into several small surface;

(3) Divide the finite element grid on all the surface. It must be carefully divided near the folding line so as to make the grid edge beside the folding line in a straight line. Then connect all grids into a continuous surface according to the actual situation of the airbag fabric, adjust the normal of all the grids towards the external airbag and adjust the unit with too small length of side, so as to reduce the time for calculation.

(4) Fold the grids according to the way of folding airbag, and make necessary adjustments to the grid.

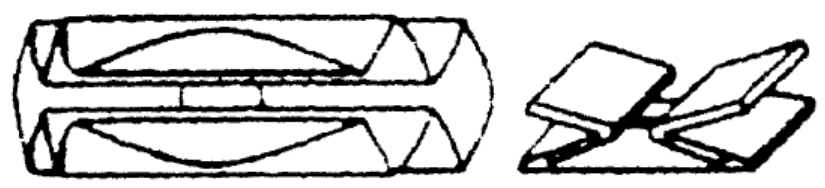

Fig. 2 the actual folding method of airbag 
The test method is to install the gas generator in a sealed container (currently use 60L container for the test), detonate gas generator and measure parameters of the gas in the container, including the pressure curve, the temperature curve and the pressure curve of the gas inside the gas generator, and then to calculate the mass flow and degree of the gas generated by the gas generator. Double pressure method and pressure temperature method are commonly used as the calculation methods.

\section{Uniform pressure model for air bag unfolding}

The uniform pressure model is a widely used airbag unfolding model at present. MADYMO, DYNA3D, APM CRASH software all use this model to calculate the process of airbag deployment. This model considers the mass flow of the gas leaked from the airbag through air vents and other factors. The air bag is regarded as the expanding control volume and the inflow and outflow of gas is calculated as the mass flow. Assuming the gas inside the control volume is the ideal gas and heat capacity coefficient is constant, and there is no heat exchange with the outside; further assumption is in control;

In the calculation of finite element, the control volume can be calculated with the following formula:

$$
V=\iiint d x d y d z=\oint x n_{x} d \Gamma \approx \sum_{i=1}^{N} \bar{x}_{i} n_{i x} A_{i}
$$

\section{Research on the experimental verification technology of airbag working performance}

After the vehicle collision accident occurs, the harm degree of passengers in the vehicle is often evaluated. According to the investigation of the accident, the most vulnerable parts of the passenger are the head and the chest 41 ], and the damage rates of the head is $4.46 \%$ while the chest $1.89 \%$. Therefore, how to evaluate the hurt on the human's head and chest in collision becomes very important. In 1960, Lissne from Wayne State University proposed Wayne hurt standard curve ['7 (]wSTc) for the impact resistance of the head in the condition of the linear acceleration and formed head injury criterion curve C.. The following formula is used:

$$
H I C=\left(t_{2}-t_{1}\right)\left[\frac{1}{t_{2}-t_{1}} \int_{t 2}^{t 1} a d t\right]^{2.5}
$$

In the formula:

1 --- any time in the process of contact;

2 --- relative to $\mathrm{t} 1$, the moment when $\mathrm{HIC}$ reached the maximum value;

A --- the acceleration of head centroid;

Because this is a transient expansion process, the airbag unfolding mode has a great effect on its performance and the change of the gas pressure inside and the final pressure value also affect the protection degree to human body greatly. The traditional air bags are generally inflated first and then expand, which presents two possibilities to hurt human body. First, a powerful forward impact force is generated when the airbag begins to expand, which does harm to the occupant who is close to the airbag module and in the direction of the expansion114,"]; second, when the airbag collides and contacts with the passenger before being unfolded completely in the process of expansion, the film 
tension generated through the continuous unfolding causes harm to the passenger's head and neck, which is shown in Fig. 4. According to the discussion above, this paper finally draws out three parameters--- acceleration, velocity and pressure as the evaluation parameters of automobile airbag performance, which are also the measurement indicators of experimental test technology put forward in this paper.

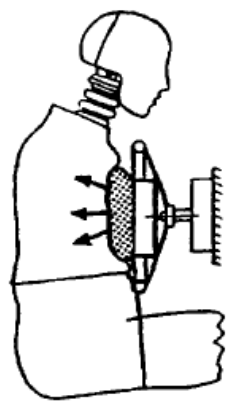

a

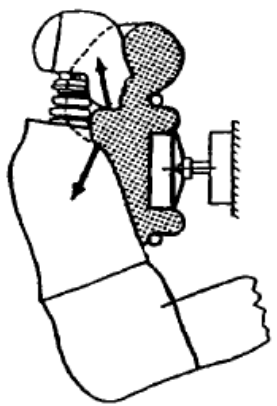

b

Fig. 4 the two kinds of harm of the airbag to the occupant (a. impact force; b. film tension)

\section{Conclusion}

This paper mainly includes the following several aspects:

1. It introduces the research contents and methods of automobile passive safety and the basic knowledge and design research methods of the airbag as a countermeasure of vehicle passive safety. A large number of literature researches show that the airbag, as a passive occupant restraint system, is very significant and effective in protect the occupant when vehicle collision occurs.

2. It introduces the computer simulation theory and key technology of the working process of automobile airbag. The computer simulation in the working process of the airbag actually is a contact problem. By studying and solving this problem, two key technologies of airbag computer simulation are proposed: airbag model and contact model. It focuses on the establishment of finite element model, simulation of airbag inflating process and contact treatment. And it applies those technologies to implement computer simulation research on the two working conditions of the airbag.

3. It has developed test bed system for the detection of the airbag system. This paper introduces the design goal of the test bed and put forwards the design requirements of the test platform to be established. Through the analysis of the airbag's protective effect on the human body, it puts forward three evaluation indicates of the airbag performance: collision acceleration, velocity and air bag gas pressure.

4. It introduces the basic theory of the test data processing and the test data preprocessing and smoothing filtering processing. The pretreatment includes noise discrimination for the acquired signal and eliminating singular term etc.. Using the theory of signal spectrum, it identifies the periodic noise mixed with the signal. The smoothing filtering processing of data uses the five point thrice smoothing filtering method with easy algorithm and better effects.

The practice shows that the application of the experimental verification technology and devices to test the simulation of automobile airbag performance, which is put forward in this paper, will be a feasible path. It provides a mean of test for the simulation method where all kinds of simulation software are applied to optimize the structure of the airbag, which also provides experimental data or corresponding criterion that are required in the future airbag computer simulation. 


\section{Acknowledgements}

This work was financially supported by the key subject building project (vehicle engineering) of Jiangxi University of Technology.

\section{References}

[1] Thomke S H. Simulation, learning and R\&D performance: Evidence from automotive development[J]. Research Policy, 1998, 27(1): 55-74.

[2] Shladover S E, Desoer C A, Hedrick J K, et al. Automated vehicle control developments in the PATH program[J]. Vehicular Technology, IEEE Transactions on, 1991, 40(1): 114-130.

[3] Marklund P O, Nilsson L. Simulation of airbag deployment using a coupled fluid-structure approach[C]//7th International LS-DYNA Users Conference. 2002.

[4] Marklund P O, Nilsson L. Simulation of airbag inflation processes using a coupled fluid structure approach[J]. Computational Mechanics, 2002, 29(4-5): 289-297.

[5] Leonardi P M. When flexible routines meet flexible technologies: Affordance, constraint, and the imbrication of human and material agencies[J]. MIS quarterly, 2011, 35(1): 147-167.

[6] Johnson A. From dynamometers to simulations: transforming brake testing technology into antilock braking systems[M]//Instrumentation between Science, State and Industry. Springer Netherlands, 2001: 199-218.

[7] Halada L, Glasa J, Weisenpacher P. Computer modelling of automobile fires[M]. INTECH Open Access Publisher, 2012.

[8] Struss P, Price C. Model-based systems in the automotive industry[J]. AI magazine, 2003, 24(4): 17.

[9] Thomke S H. Managing experimentation in the design of new products[J]. Management Science, 1998, 44(6): 743-762.

[10] Thomke S, Fujimoto T. The Effect of "Front - Loading" Problem - Solving on Product Development Performance[J]. Journal of product innovation management, 2000, 17(2): 128-142. 